\title{
HARMONIES IN NATURE: A DIALOGUE BETWEEN MATHEMATICS AND PHYSICS
}

\author{
Man-Keung SIU \\ Department of Mathematics \\ The University of Hong Kong \\ Pokfulam, Hong Kong \\ mathsiu@hkucc.hku.hk
}

\begin{abstract}
The customary practice in school to teach mathematics and physics as two separate subjects has its grounds. However, such a practice deprives students of the opportunity to see how the two subjects are intimately interwoven. This paper discusses the design and implementation of an enrichment course for school pupils in senior secondary school who are about to embark on their undergraduate study. The course tries to integrate the two subjects with a historical perspective.
\end{abstract}

\section{Why is an enrichment course on mathematics-physics de- signed?}

In school it is a customary practice to teach mathematics and physics as two separate subjects. In fact, mathematics is taught throughout the school years from primary school to secondary school, while physics, as a full subject on its own, usually starts in senior secondary school. This usual practice of teaching mathematics and physics as two separate subjects has its grounds. To go deep into either subject one needs to spend at least a certain amount of class hours, and to really understand physics one needs to have a sufficiently prepared background in mathematics. However, such a practice deprives students of the opportunity to see how the two subjects are intimately interwoven. Indeed, in past history there was no clear-cut distinction between a scientist, not to mention so specific as a physicist, and a mathematician.

Guided by this thought we try to design an enrichment course for school pupils in senior secondary school, who are about to embark on their undergraduate study in two to three years' time, that tries to integrate the two subjects with a historical perspective. Conducting it as an enrichment course, we are free from an examination-oriented teaching-learning environment and have much more flexibility with the content. Admittedly, this is not exactly the same as the normal classroom situation with the constraint imposed by an official syllabus and the pressure exerted by a public examination. However, just like building a mathematical model, we like to explore what happens if we can have a bit more freedom to do things in a way we feel is nearer to our ideal.

Albert Einstein and Leopold Infeld sum up the situation succinctly, "In the whole history of science from Greek philosophy to modern physics there have been constant attempts to reduce the apparent complexity of natural phenomena to some simple fundamental ideas and relations. This is the underlying principle of all natural philosophy." [Einstein \& Infeld, 1938]. Such a process makes demand on one's curiosity and imagination, but at the same time requires disciplined and critical thinking. Precision in mathematics as well as in words is called for. Galileo Galilei already referred to mathematics as the language of science in his Il Saggiatore (The Assayer) of 1623, "Philosophy 
is written in this grand book - I mean the universe - which stands continually open to our gaze, but it cannot be understood unless one first learns to comprehend the language and interpret the characters in which it is written. It is written in the language of mathematics, and its characters are triangles, circles, and other geometric figures, without which it is humanly impossible to understand a single word of it; without these, one is wandering about in a dark labyrinth."

By promoting this view Galileo made a significant step forward in switching the focus from trying to answer "why" to trying to answer "how (much)", that is, from a qualitative aspect to a quantitative aspect. In the Eastern world a similar sentiment was expressed by many authors of ancient classics that may sound like bordering on the mystical side. One such typical example is found in the preface of Sun Zi Suan Jing (Master Sun's Mathematical Manual) in the 4th century, "Master Sun says: Mathematics governs the length and breadth of the heavens and the earth; affects the lives of all creatures; forms the alpha and omega of the five constant virtues; acts as the parents for yin and yang; establishes the symbols for the stars and the constellations; manifests the dimensions of the three luminous bodies; maintains the balance of the five phases; regulates the beginning and the end of the four seasons; formulates the origin of myriad things; and determines the principles of the six arts."

The conviction in seeing beauty and order in Nature was long-standing. Plato's association of the five regular polyhedra to the theory of four elements in Timaeus (c.4th century B.C.) is an illustrative example. Over a millennium later, Johannes Kepler tried to fit in the motion of the six known planets (Saturn, Jupiter, Mars, Earth, Venus, Mercury) in his days with the five regular polyhedra in Mysterium Cosmographicum of 1596. By calculating the radii of inscribed and circumscribed spheres of the five regular polyhedra nestled in the order of a cube, a tetrahedron, a dodecahedron, an icosahedron and an octahedron, he obtained results that agreed with observed data to within $5 \%$ accuracy! He also thought that he had explained why there were six planets and not more! Now we realize the lack of physical ground in his theory, beautiful as it may seem. Still, it is a remarkable attempt to associate mathematics with physics, and indeed it led to something fruitful in the subsequent work of Kepler.

Well into the modern era the explanatory power of mathematics on Nature is still seen by many to be mystical but fortunate. Eugene Paul Wigner, 1963 Nobel Laureate in physics, refers to it as "the unreasonable effectiveness of mathematics in the natural sciences". Heinrich Rudolf Hertz even said (referring to the Maxwell's equations which predicted the presence of electromagnetic wave that he detected in the laboratory in 1888.), "One cannot escape the feeling that these mathematical formulas have an independent existence of their own, that they are wiser than we are, wiser even than their discoverers, that we get more out of them than was originally put into them." Robert Mills, an eminent physicists of the Yang-Mills gauge theory fame, says, "You can't hope to understand the [physics / math] until you've understood the [math / physics]." [Mills, 1994]. This dictum that emphasizes a two-way relationship between mathematics and physics furnishes the guideline for our enrichment course.

\section{How is such a course run?}

The enrichment course, with its title same as that of this paper, ran for ten sessions each taking up three hours on a weekend (outside of the normal school hours). It had been run twice, in the spring of 2006 and 2007, in collaboration with a colleague 
at the Department of Physics in my university. Much as we wish to offer a truly integrated course, other constraints and factors (individual expertise, affordable time of preparation, inadequacy on our part, lack of experience in this new venture) force some sort of division of labour so that each one of us took up about half of the course. However, we still tried to maintain a spirit of integration in having a balanced emphasis on the mathematics and the physics in a suitable manner. In this paper I will naturally tell more about the part I took up, which involved the first two sessions, two intermittent sessions and the final session.

The underlying theme of the course is the role and evolution of mathematics, mainly geometry and calculus, with related topics in linear algebra, in an attempt to understand the physical world, from the era of Isaac Newton to that of James Clerk Maxwell and beyond it to that of Albert Einstein. In other words, it tries to tell the story of triumph in mathematics and physics in the past four centuries. The physics provides both the source of motivation and the applications of a number of important topics in mathematics. Along the way both ideas and methods are stressed, to be learnt in an interactive manner through discussion in tutorials and group work on homework assignments. A rough sketch of the content of the course is summarized in Table 1. Considering the level of the course, it is to be expected that topics near to the end are treated only after a fashion, mainly for broadening the vista of the students rather than for teaching them the technical details.

\section{Table 1}

\section{A sketch of the content of the course}

Each session of the enrichment course consists of a lecture in the first hour followed by a tutorial. The lecture serves to highlight some keypoints and outline the development of the topic. What is covered is selective in the sense that the material illustrates some theme rather than provides a comprehensive account. Interested students are advised to read up on their own relevant references suggested in each session. [A selected sample of such books can be found in the list of references, some of which are more suitable for the teacher than the student (Barnett, 1949; Boyer, 1968; Einstein \& Infeld, 1938; Feynman, 1995; Hewitt, 2006; Lines, 1994; Longair, 1984; Mills, 1994; Olenik, Apostol \& Goldstein, 1985/1986; Pólya, 1963; Siu, 1993).] The course is seen as a means to arouse, to foster and to maintain the enthusiasm of students in mathematics and physics more than as a means to equip them with a load of knowledge.

To keep within the prescribed length of the paper I would not give a full account of the content but select certain parts, particularly the beginning part that sets the tone of the course, with supplementary commentary, to illustrate the intent of the enrichment course. The intent is to highlight the beautiful (some would say uncanny!) and intimate relationship between mathematics and physics, in many cases even mathematical ideas that have lain quietly in waiting for many years (sometimes more than a thousand years!) that enhance theoretical understanding of physical phenomena. In fact the relationship is two-way so that the two subjects benefit mutually from each other in their development. In section 4 some sample problems in tutorials are appended in the hope of better illustrating this intention.

The course begins with a discussion on the Aristotelian view of the physical world that came to be known since the 4th century B.C.. All terrestrial matters, which are 
held to be different from heavenly matters, are believed to contain a mixture of the four elements in various compositions. Each of the four elements is believed to occupy a natural place in the terrestrial region, in the order of earth (lowest), water, air, fire (uppermost). Left to itself, the natural motion of an object is to go towards its natural position, depending on the composition and the initial position. Hence, a stone (earth) falls to the ground but a flame (fire) goes up in the air. A natural motion has a cause. It is believed that the weight of a stone is the cause for its free falling motion. According to the Aristotelian view, a heavier stone will fall faster than a lighter one. Any motion that is not a natural motion is called a violent motion, believed to be caused by a force.

We next bring in the physical world view that Galileo propounded in the first part of the 17th century. In particular, he demolished the theory that a heavier object falls faster by mathematical reasoning (thought-experiment) in Discorsi e dimonstrazioni matematiche intorno a due nuove scienze (Discourses and Mathematical Demonstrations Concerning Two New Sciences) of 1638. Suppose object $A_{1}$ has a larger weight $W_{1}$ than the weight $W_{2}$ of object $A_{2}$. Tie the objects $A_{1}$ and $A_{2}$ together to form an object of weight $W_{1}+W_{2}$. The more rapid one will be partly retarded by the slower; the slower one will be somewhat hastened by the swifter. Hence, the united object will fall slower than $A_{1}$ alone but faster than $A_{2}$ alone. However, the united object, being heavier than $A_{1}$, should fall faster than $A_{1}$ alone. This is a contradiction! [Hawking, 2002, p.446]. A commonly told story says that Galileo dropped two balls of different weights from the top of the Tower of Pisa to arrive at his conclusion. There is no historical evidence that he actually did that. The significant point does not lie so much in whether Galileo actually carried out the experiment but in his arrival at the conclusion by pure reasoning. Together with pure reasoning, Galileo was known for his emphasis on observations and experiments as well, notably his experiments with an inclined plane. By observing that a ball rolling down an inclined plane will travel up another inclined plane joined to the first one at the bottom until it reaches the same height, he saw that the ball will travel a greater distance if the second inclined plane is placed less steep than the first one, the greater if the second inclined plane is less steep. From thence a thought-experiment comes in again. If the second inclined plane is actually placed in a horizontal position, the ball will travel forever without stopping. "Furthermore we may remark that any velocity once imparted to a moving body will be rigidly maintained as long as the external causes of acceleration or retardation are removed, a condition which is found only on horizontal planes. ... it follows that motion along a horizontal plane is perpetual ..." [Hawking, 2002, p.564]. This motivated him to announce his famous law of inertia, which becomes the first law of motion in Newton's Philosophiae naturalis principia mathematicas (Mathematical Principles of Natural Philosophy) of 1687: "Every body persevers in its state of rest, or of uniform motion in a right line, unless it is compelled to change that state by forces impressed thereon." [Hawking, 2002, p.743]. This fundamental modification on the Aristotelian view (in a sense actually more natural according to daily experience!) that a force acting on an object is exemplified not by the speed of its motion but by the change in speed (acceleration), led to a quantitative description of this relationship in Newton's second law of motion (which yields the famous formula $F=m a$ ). It turned a new page in the development of physics. We follow with a discussion on the work of Johannes Kepler in calculating the orbit of Mars based on the meticulously kept observed data of Tycho Brahe [Koestler, 1959]. On the one hand the story displays a beautiful interplay between theory and 
experiment. On the other hand Kepler's laws on planetary motion provide a nice lead to a discussion on Newton's law of universal gravitation.

We next discuss the theory of wave motion along with the mathematics, culminating in the theory of electromagnetism and Maxwell's equations. Mathematics owed to physics a great debt in that a large part of mathematical analysis that was developed in the 18th and 19th centuries have to do with the Vibrating String Problem. We talk about the all-important notions of function and of equation. Together with the discussion on vector calculus and the generalized Fundamental Theorem of Calculus, there is much more material than enough to take up the second part of the course. The unification of electricity, magnetism and light through the electromagnetic wave is a natural lead into the final third of the course, which is spent on a sketch of the theory of relativity and on quantum mechanics. Some probability theory is introduced to let students appreciate the stochastic aspect that is not usually encountered in the usual school curriculum. The close relationship between geometry and physics is stressed in the final episode on the theory of general relativity. In a letter to Arnold Sommerfeld dated October 29, 1912 (collected in A. Hermann, Einstein/Sommerfeld Briefwechsel, Schwabe Verlag, Stuttgart, 1968, p.26) Albert Einstein wrote, "I am now exclusively occupied with the problem of gravitation, and hope, with the help of a local mathematician friend, to overcome all the difficulties. One thing is certain, however, that never in my life have I been quite so tormented. A great respect for mathematicians has been instilled within me, the subtler aspects of which, in my stupidity, I regarded until now as pure luxury. Against this problem, the original problem of the theory of relativity is child's play." The "mathematician friend' refers to Einstein's school friend Marcel Grossmann, and the mathematics refers to Riemannian geometry and tensor calculus. The story on the work of Carl Friedrich Gauss and Georg Friedrich Bernhard Riemann in revealing the essence of curvature which lies at the root of the controversy over the Fifth Postulate in Euclid's Elements (but which had been masked for more than two thousand years when the attention of mathematicians was directed into a different direction) and its relation to Einstein's idea on gravitation theory is fascinating for both mathematics and physics. No wonder Riemann concluded his famous 1854 lecture titled Über die Hypothesen welche der Geometrie zu Grunde liegen (On the hypotheses which lie at the foundation of geometry (an English translation can be found in David Eugene Smith (ed.), A Source Book in Mathematics, McGraw-Hill, New York, 1929, pp.411-425) with: "This path leads out into the domain of another science, into the realm of physics, into which the nature of this present occasion forbids us to penetrate."

\section{Some sample problems in tutorials}

In this course more than half of the time in each session is spent as a tutorial, which is regarded as an integral part of the learning experience. Students work in small groups with guidance or hint provided on the side by the teacher and a team of (four) teaching assistants. At the end of each session there is a guided discussion with presentations by students. A more detailed record of the solution is put on the web afterward for those who are interested to probe further. Some sample problems in the tutorials are given below to convey a flavour of the workshop.

Question 1. $A, B, C, D$ move on straight lines on a plane with constant speeds. (The speed of each chap may be different from that of another.) It is known that each of $A$ and $B$ meets the other three chaps at distinct points. Must $C$ and $D$ meet? 
Under what condition will the answer be 'yes' (or 'no')?

Discussion: $C$ and $D$ will (respectively will not) meet if they do not move (respectively move) in the same or opposite directions. The catch is a commonly mistaken first reaction to draw a picture with two straight lines emanating from a common point $M_{A B}$ (the point where $A$ and $B$ meet) and two more straight lines, one intersecting the first line at $M_{A C}$ and the second line at $M_{B C}$, the other one intersecting the first line at $M_{A D}$ and the second line at $M_{B D}$. It seems that the answer comes out obviously from the picture until one realizes that a geometric intersecting point needs not be a physical intersecting point! This problem is set as the first problem in the first tutorial to lead the class onto the important notion of spacetime, which will feature prominently in the theory of relativity. Viewed in this context, no calculation is needed at all!

Question 2. Suppose you only know how to calculate the area of a rectangle our ancestors started with that. Explain how you would calculate the area of a triangle by approximating it with many many rectangles of very small width. This answer, by itself, does not sound too exciting. You can obtain it by other means, for instance by dissection - our ancestors did just that! However, what is exciting is the underlying principle that can be adapted to calculate the area of regions of other shapes. Try to carry out a similar procedure for a parabolic segment. (Find the area under the curve given by $y=k x^{2}$ from $x=0$ to $x=a$. What happens if you are asked to find the area under the curve $y=k x^{3}$ ? $y=k x^{4}$ ? ‥? Later you will see how a result enables us to solve this kind of problem in a uniform manner.)

Discussion: This problem is set at the beginning of the course to introduce some ideas and methods devised by ancient Greeks and ancient Chinese on problems in quadrature, to be contrasted with the power of calculus developed during the 17th and 18th centuries, culminating in the Fundamental Theorem of Calculus with its generalized form (Stokes' Theorem) established through the development of the theory of electromagnetism in the 19th century. For this particular problem some clever formulae on the sum of consecutive $r$ th power of integers $1^{r}+2^{r}+3^{r}+\cdots+N^{r}$ are needed. That kind of calculation is not totally foreign to the experience of school pupils and yet offers some challenge beyond what they are accustomed to, which is therefore of the level of difficulty the workshop is gauged at. After struggling with specific but seemingly ad hoc 'tricks' of this sort, students would appreciate better the power afforded by the Fundamental Theorem of Calculus when they learn it later.

Question 3. (a) By computing the sum

$$
1+z+z^{2}+\cdots+z^{n}
$$

where $z=e^{i \theta}$, and using Euler's formula

$$
e^{i \theta}=\cos \theta+i \sin \theta
$$

find a simple expression for

and

$$
\begin{array}{r}
1+\cos \theta+\cos 2 \theta+\cdots+\cos n \theta \\
\sin \theta+\sin 2 \theta+\cdots+\sin n \theta .
\end{array}
$$

(b) Apply the result in (a) to calculate the area under the curve $y=\sin x$ on $[0, \pi]$ from scratch in the way you did for $y=x^{2}$ in the first tutorial. Do the same for $y=\cos x$ on $[0, \pi]$. (How do you normally calculate this area in your class at school?)

Discussion: Besides introducing a most beautiful formula in mathematics, this problem further strengthens students' appreciation of the Fundamental Theorem of 
Calculus. In the course of explaining Euler's formula students are led into the realm of complex numbers, to the 'twin' functions of logarithm and exponentiation.

Question 4. (a) Pierre Simon Laplace (1749-1827) once said, "By shortening the labors, the invention of logarithms doubled the life of the astronomer." To appreciate this quotation, let us work on an multiplication problem $(81276 \times 96343)$ like people did before the invention of logarithm. The method, known as "prosthaphaeresis", is based on the addition formula of trigonometric functions.

(i) If $2 \cos A=0.81276$ and $\cos B=0.96343$, find $A$ and $B$.

(ii) Calculate $A+B, A-B$, and hence calculate $\cos (A+B), \cos (A-B)$.

(iii) Calculate $\cos (A+B)+\cos (A-B)$, which is equal to $2 \cos A \cos B$, and hence find out what $81276 \times 96343$ is.

(b) Compare Napier's logarithm with the natural logarithm you learn in school.

(c) Making use of the idea Leonhard Euler (1707-1783) explained in Chapter XXII of his Vollständige Anleitung zur Algebra (1770), compute the natural logarithm of 5, ln 5, in the following steps:

(i) As 5 lies between 1 and 10, so $\ln 5$ lies between 0 and 1. Take the average of 0 and 1 , which is $1 / 2$. Compute $10^{1 / 2}$, which is the square root of 10 , say $a_{1}$.

(ii) Decide whether 5 falls into $\left[1, a_{1}\right]$ or $\left[a_{1}, 10\right]$. Hence decide whether $\ell n 5$ falls into $[0,1 / 2]$ or $[1 / 2,1]$. It turns out en 5 falls into $[1 / 2,1]$. Take the average of $1 / 2$ and 1 , which is $3 / 4$. Compute $10^{3 / 4}$, which is the square root of 10 multiplied by the square root of $10^{1 / 2}$, say $a_{2}$.

(iii) Decide whether 5 falls into $\left[a_{1}, a_{2}\right]$ or $\left[a_{2}, 10\right]$. Hence decide whether $\ln 5$ falls into $[1 / 2,3 / 4]$ or $[3 / 4,1]$. It turns out $\ell n 5$ falls into $[1 / 2,3 / 4]$. Take the average of $1 / 2$ and $3 / 4$, which is $5 / 8$. Compute $10^{5 / 8}$, which is the square root of $10^{1 / 2}$ multiplied by the square root of $10^{3 / 4}$, say $a_{3}$.

(iv) Continue with the algorithm until you reach a value of $\ell n 5$ accurate to three decimal places.

Discussion: Note the similar underlying idea of converting multiplication to addition in "prosthapharesis" and in logarithm. That allows the class to see how John Napier and later Henry Briggs devised their logarithm in the early 17th century. The bisection algorithm explained in (c), though seemingly cumbersome from a modern viewpoint, is nonetheless very natural and simple, reducing the calculation to only finding square root. It provides an opportunity to go into the computation of square root by the ancients, first propounded in detail in the ancient Chinese classics Jiu Zhang Suan Shu (Nine Chapters on the Mathematical Art) compiled between 100 B.C. and 100 A.D. For the generation of youngsters who grow up with calculators and computers, this kind of 'old' techniques may add a bit of amazement as well as deeper comprehension.

Question 5. In an $x-t$ spacetime diagram drawn by an observer $S$ who regards himself as stationary, draw the world-line for $S$ and the world-line for an observer $S^{\prime}$ moving with uniform velocity $v$ (relative to $S$ ). At $t=0$ both $S$ and $S^{\prime}$ are at the 
origin $O$. Both $S$ and $S^{\prime}$ observe a light signal sent out from $O$ at $t=0$, reflected back by a mirror at a point $P$, then received by $S^{\prime}$ at $Q$. Which point on the world-line for $S^{\prime}$ will $S^{\prime}$ regard as an event simultaneous with the reflection of the light signal at $P$ ? Call this point $P^{\prime}$. Show that the slope of the line $P^{\prime} P$ is equal to $v / c^{2}$, where $c$ is the speed of light (units omitted). [The physical interpretation is as follows. $S$ regards two events, perceived as simultaneous by $S^{\prime}$, as separated by a time $\Delta t$ given by $\Delta t=\left(v / c^{2}\right) \Delta x$, where $\Delta x$ is the distance between the events measured by $S$ and $v$ is the velocity of $S^{\prime}$ relative to $S$.]

Discussion: We pay attention to the physical interpretation of a mathematical calculation and vice versa. This problem focuses on the key notion of simultaneity in the theory of special relativity. There is a note of caution for this problem. The picture of the spacetime diagram (according to the observer $S$ ) is to be seen in two ways: (i) the picture as it is, just like a picture one is accustomed to see in school geometry, (ii) the coordinate system of $S$ with coordinates assigned to each event. In the lecture we take good care in denoting points in (i) by letters $P, Q, P^{\prime}, O$, etc., and events in (ii) by $(x(P), t(P)),(x(Q), t(Q)),\left(x\left(P^{\prime}\right), t\left(P^{\prime}\right)\right),(x(O), t(O))$, etc. One can read the same in the shoes of the other observer $S^{\prime}$, in which case events in (ii) will be denoted by $\left(x^{\prime}(P), t^{\prime}(P)\right),\left(x^{\prime}(Q), t^{\prime}(Q)\right),\left(x^{\prime}\left(P^{\prime}\right), t^{\prime}\left(P^{\prime}\right)\right),\left(x^{\prime}(O), t^{\prime}(O)\right)$, etc. In the lecture we also explain how $x(P), t(P)$ are related to $x^{\prime}(P), t^{\prime}(P)$ and vice versa (by the Lorentz transformation).

\section{Conclusion}

The triumph of Maxwell's theory on electromagnetism resolved many problems and yet introduced new difficulties that were resolved by Einstein's theory of special relativity. The triumph of Einstein's theory of special relativity resolved many problems and yet introduced new difficulties that were resolved by Einstein's theory of general relativity. But then the theory of general relativity introduces a more difficult problem on incompatibility with quantum mechanics, which is not revealed until one comes up with a situation where both the mass involved is very large and the size involved is very small, for instance, a black hole [Greene, 1999; Penrose, 2004]. Physics will march on to solve further problems, and so will mathematics, hand-in-hand with physics, in a harmonious way.

\section{REFERENCES}

- Barnett, L., 1949, The Universe and Dr. Einstein, London: Victor Gollancz.

- Boyer, C., 1968, A History in Mathematics, New York: Wiley (revised by U.C. Merzbach, 2nd edition, 1989).

- Einstein, A., Infeld, L., 1938, The Evolution of Physics: The Growth of Ideas From Early Concepts to Relativity and Quanta, Cambridge: Cambridge University Press (2nd edition, 1961).

- Feynman, R.P., 1995, Six Easy Pieces: Essentials of Physics Explained by its most Brilliant Teacher, Reading: Helix Books (selected chapters from The Feynman Lectures on Physics, Volume 1-3, R.P. Feynman, Reading: Addison-Wesley, 1963-1965).

- Greene, B., 1999, The Elegant Universe: Superstrings, Hidden Dimensions, and the Quest of the Ultimate Theory, New York: W.W. Norton. 
- Hawking, S. (ed.), 2002, On the Shoulders of Giants: The Great Works of Physics and Astronomy, London-Philadelphia: Running Press.

- Hewitt, P.G., 2006, Conceptual Physics, 10th edition, San Francisco: Benjamin Cummings.

- Koestler, A., 1959, The Sleepwalkers: A History of Man's Changing Vision of the Universe, London: Hutchinson.

- Lines, M.E., 1994, On the Shoulders of Giants, Bristol-Philadelphia: Institute of Physics Publishing.

- Longair, M.S., 1984, Theoretical Concepts in Physics: An Alternative View of Theoretical Reasoning in Physics, Cambridge: Cambridge University Press (2nd edition, 2003).

- Mills, R., 1994, Space, Time and Quanta: An Introduction to Contemporary Physics, New York: W.H. Freeman.

- Olenik, R.P., Apostol, T.M., Goldstein, D.L., 1985, The Mechanical Universe: Introduction to Mechanics and Heat, Cambridge: Cambridge University Press.

- Olenik, R.P., Apostol, T.M., Goldstein, D.L., 1986, Beyond the Mechanical Universe: From Electricity to Modern Physics, Cambridge: Cambridge University Press.

- Penrose, R., 2004, The Road to Reality: A Complete Guide to the Laws of the Universe, London: Vintage Books.

- Pólya, G., 1963, Mathematical Methods in Science, Washington, D.C.: Mathematical Association of America (reprinted in 1977).

- Siu, M.K., 1993, 1,2,3,... and Beyond (in Chinese), Hong Kong: Joint Publishing (HK). 


\begin{tabular}{|c|c|c|}
\hline Time period & Physics & Mathematics (mainly) \\
\hline 4th century B.C. & Physical view of Aristotle & Euclidean geometry \\
\hline $\begin{array}{l}\text { many centuries } \\
\text { in between }\end{array}$ & & $\begin{array}{l}\text { geometry (area / volume) } \\
\text { algebra (equations) }\end{array}$ \\
\hline 17th century & $\begin{array}{l}\text { physical view } \\
\text { of Copernicus, } \\
\text { Kepler, Galileo, } \\
\text { Newton, ... }\end{array}$ & $\begin{array}{l}\text { vectors in } \mathbb{R}^{2} \text { and } \mathbb{R}^{3} \text {, calculus } \\
\text { in one variable (functions, } \\
\text { including polynomial, } \\
\text { rational, trigonometric, } \\
\text { logarithmic and exponential) }\end{array}$ \\
\hline 18th century & wave and particle & $\begin{array}{l}\text { differential equations, } \\
\text { Fourier analysis, complex numbers }\end{array}$ \\
\hline 19th century & $\begin{array}{l}\text { theory of } \\
\text { electromagnetism } \\
\text { (Maxwell's equations) }\end{array}$ & $\begin{array}{l}\text { vector calculus, Stokes' Theorem } \\
\text { (Fundamental Theorem } \\
\text { of Calculus) }\end{array}$ \\
\hline 20th century & $\begin{array}{l}\text { theory of special } \\
\text { and general relativity, } \\
\text { quantum mechanics }\end{array}$ & $\begin{array}{l}\text { probability theory, } \\
\text { non-Euclidean } \\
\text { geometries of spacetime }\end{array}$ \\
\hline
\end{tabular}

Table 1 